\title{
MOMENT PROBLEMS FOR COMPACT SETS
}

\author{
J. D. CHANDLER, JR.
}

(Communicated by Richard R. Goldberg)

\begin{abstract}
The solvability of the Hausdorff moment problem for an arbitrary compact subset of Euclidean $n$-space is shown to be equivalent to the nonnegativity of a family of quadratic forms derived from the given moment sequence and the given compact set. A variant theorem for the one-dimensional case and an analogous theorem for the trigonometric moment problem are also given. The one-dimensional theorems are similar to theorems of Krein and Nudel'man [11], but the proofs, unlike those in [11], do not depend on the existence of a standard form for polynomials which are nonnegative on a given compact set.
\end{abstract}

1. Let $n$ be a positive integer, $R$ the real line, and $N$ the set of all nonnegative integers. Let $R^{n}$ be Euclidean $n$-space, i.e. the set of all $n$-tuples $x=\left(x_{1}, x_{2}, \ldots, x_{n}\right)$ of real numbers; and let $N^{n}$ be the set of all $n$-tuples $j=\left(j_{1}, j_{2}, \ldots, j_{n}\right)$ of nonnegative integers. The elements of $N^{n}$ will be called multi-indices. We shall often write $x^{j}$ for the product

$$
x_{1}^{j_{1}} x_{2}^{j_{2}} \cdots x_{n}^{j_{n}}
$$

where $x \in R^{n}$ and $j \in N^{n}$.

In this section we shall solve the following $n$-dimensional generalization of the Hausdorff moment problem $[\mathbf{9}, \mathbf{1 1}]$ : given a compact subset $K$ of $R^{n}$ and a multiply indexed sequence of real numbers

$$
\left\{c_{j}: j \in N^{n}\right\}=\left\{c\left(j_{1}, j_{2}, \ldots, j_{n}\right):\left(j_{1}, j_{2}, \ldots, j_{n}\right) \in N^{n}\right\}
$$

find necessary and sufficient conditions that there exist a positive Borel measure $\mu$, supported on $K$, such that $c_{j}=\int_{K} x^{j} d \mu(x)$, i.e.

$$
c\left(j_{1}, j_{2}, \ldots, j_{n}\right)=\int_{K} x_{1}^{j_{1}} x_{2}^{j_{2}} \cdots x_{n}^{j_{n}} d \mu(x)
$$

for every multi-index $j ; \mu$ is supported on $K$ if $\mu(E)=\mu(E \cap K)$ for every Borel subset $E$ of $R^{n}$. Our solution will be that certain quadratic forms are nonnegative; the quadratic forms are determined by the "moment sequence" $\left\{c_{j}: j \in N^{n}\right\}$ and by a decomposition of the compact set $K$.

In order to state our solution, we must first describe the decomposition of $K$, which is not unique. Let

$$
[a, b]=\left[a_{1}, b_{1}\right] \times\left[a_{2}, b_{2}\right] \times \cdot \times\left[a_{n}, b_{n}\right]
$$

be a rectangle containing $K$, where $a_{1} \leq b_{1}, a_{2} \leq b_{2}, \ldots, a_{n} \leq b_{n}$, and $a=$ $\left(a_{1}, a_{2}, \ldots, a_{n}\right), b=\left(b_{1}, b_{2}, \ldots, b_{n}\right)$. Since the topology of $R^{n}$ has a countable

Received by the editors August 12, 1987.

1980 Mathematics Subject Classification (1985 Revision). Primary 44A60, 42A70; Secondary 47B15. 
basis, there exists an at most countable family of open balls $\left\{B\left(w, r_{w}\right): w \in W\right\}$ such that

$$
K=[a, b] \backslash\left(\bigcup_{w \in W} B\left(w, r_{w}\right)\right) .
$$

Here $B(w, r)$ is the open ball in $R^{n}$ with center $w=\left(w_{1}, w_{2}, \ldots, w_{n}\right)$ and radius $r$.

Given the decomposition (1) of $K$, we can describe the quadratic forms which will appear in our solution of the moment problem. For $i=1,2, \ldots, n$, let $e_{i}$ be the element of $R^{n}$ whose $i$ th component is 1 and whose other components are all 0. Then

$$
\begin{aligned}
c_{j+e_{i}} & =c\left(j_{1}, \ldots, j_{i-1}, j_{i}+1, j_{i+1}, \ldots, j_{n}\right), \\
c_{j+2 e_{i}} & =c\left(j_{1}, \ldots, j_{i-1}, j_{i}+2, j_{i+1}, \ldots, j_{n}\right),
\end{aligned}
$$

for $j \in N^{n}$. For each $w \in W$, let

$$
\begin{gathered}
c_{j}^{w}=\sum_{i=1}^{n}\left(c_{j+2 e_{i}}-2 w_{i} c_{j+e_{i}}+w_{i}^{2} c_{j}\right)-r_{w}^{2}, \quad j \in N^{n}, \\
P_{w}(x)=\|x-w\|^{2}-r_{w}^{2}=\sum_{i=1}^{n}\left(x_{i}-w_{i}\right)^{2}-r_{w}^{2}, \quad x \in R^{n} .
\end{gathered}
$$

Note that $B\left(w, r_{w}\right)$ is the set of all points $x$ in $R^{n}$ for which $P_{w}(x)$ is negative. The idea of using the polynomials $P_{w}$ to describe the "holes" of $B\left(w, r_{w}\right)$ of $K$ was derived from [7] and [11]; see also [4].

THEOREM 1. Let $K$ be a compact subset of $R^{n}$ and let $\left\{c_{j}: j \in N^{n}\right\}$ be a multiply indexed sequence of real numbers. Then a necessary and sufficient condition that there exist a positive Borel measure $\mu$ supported on $K$ such that

$$
c_{j}=\int_{K} x^{j} d \mu(x), \quad j \in N^{n},
$$

is that, for every multiply indexed sequence of complex numbers $\left\{\zeta_{j}: j \in N^{n}\right\}$ with only finitely many nonzero terms,

(i) $\sum c_{j+k} \zeta_{j} \bar{\zeta}_{k} \geq 0$,

(ii) for $i=1,2, \ldots, n$,

$$
a_{i} \cdot \sum c_{j+k} \zeta_{j} \bar{\zeta}_{k} \leq \sum c_{j+k+e_{i}} \zeta_{j} \bar{\zeta}_{k} \leq b_{i} \cdot \sum c_{j+k} \zeta_{j} \bar{\zeta}_{k}
$$

(iii) for every $w \in W, \sum c_{j+k}^{w} \zeta_{j} \bar{\zeta}_{k} \geq 0$.

In the one-dimensional case, conditions (i) and (ii) constitute a well-known criterion for the solvability of the Hausdorff moment problem for the compact interval $[a, b]\left[11\right.$, p. 64]. ( $\sum$ extends over all $j, k$ in $N^{n}$.)

PROOF OF NECESSITY. Suppose that there exists a positive Borel measure $\mu$ supported on $K$ such that (2) holds. Then

$$
\sum c_{j+k} \zeta_{j} \bar{\zeta}_{k}=\int_{K}\left|\sum \zeta_{j} x^{j}\right|^{2} d \mu(x),
$$


so that (i) holds, and (ii) follows from the inequalities

$$
\begin{aligned}
a_{i} \cdot \int_{K}\left|\sum \varsigma_{j} x^{j}\right|^{2} d \mu(x) & \leq \int_{K} x_{i} \cdot\left|\sum \varsigma_{j} x^{j}\right|^{2} d \mu(x) \\
& \leq b_{i} \cdot \int_{K}\left|\sum \varsigma_{j} x^{j}\right|^{2} d \mu(x), \text { for } i=1,2, \ldots, n .
\end{aligned}
$$

Since $P_{w}(x)>0$ for $x \in K$, we have that

$$
\sum c_{j+k}^{w} \varsigma_{j} \bar{\zeta}_{k}=\int_{K} P_{w}(x) \cdot\left|\sum \zeta_{j} x^{j}\right|^{2} d \mu(x) \geq 0
$$

for $w \in W$, so that (iii) holds. Q.E.D.

PROOF OF SUFFICIENCY. Suppose that conditions (i)-(iii) hold, and define the functions $K_{j}\left(j \in N^{n}\right)$ by $K_{j}(k)=c_{j+k}$ for $j, k \in N^{n}$. Let $\mathscr{H}_{0}$ be the complex-linear span of the family of functions $\left\{K_{j}: j \in N^{n}\right\}$. Let $f=\sum \varsigma_{j} K_{j}$ and $g=\sum \omega_{j} K_{j}$ belong to $\mathscr{K}_{0}$, where the "sequences" $\left\{\varsigma_{j}: j \in N^{n}\right\}$ and $\left\{\omega_{j}: j \in N^{n}\right\}$ have only finitely many nonzero terms. Then

$$
\begin{aligned}
& \sum_{j, k} \varsigma_{j} \bar{\omega}_{k} c_{j+k}=\sum_{j, k} \varsigma_{j} \bar{\omega}_{k} K_{j}(k)=\sum_{k} \bar{\omega}_{k} \sum_{j} \varsigma_{j} K_{j}(k)=\sum_{k} \bar{\omega}_{k} f(k), \\
& \sum_{j, k} \varsigma_{j} \bar{\omega}_{k} c_{j+k}=\sum_{j, k} \varsigma_{j} \bar{\omega}_{k} K_{k}(j)=\sum_{j} \varsigma_{j} \sum_{k} \bar{\omega}_{k} \overline{K_{k}(j)}=\sum_{j} \varsigma_{j} \overline{g(k)},
\end{aligned}
$$

so that $\sum_{j, k} \varsigma_{j} \bar{\omega}_{k} c_{j+k}$ depends only on the functions $f$ and $g$ and not upon the decompositions $f=\sum \varsigma_{j} K_{j}, g=\sum \omega_{j} K_{j}$. Thus condition (i) implies that the equation

$$
\langle f, g\rangle=\sum_{j, k} \varsigma_{j} \bar{\omega}_{k} c_{j+k}, \quad f, g \in \mathscr{H}_{0}
$$

defines an inner product $\langle\cdot, \cdot\rangle$ on $\mathscr{H}_{0}$.

Now define the linear transformations $A_{1}, A_{2}, \ldots, A_{n}$ on $\mathscr{L}_{0}$ by

$$
\left(A_{i} f\right)(j)=f\left(j+e_{i}\right), \quad f \in \mathscr{L}_{0}, j \in N^{n}
$$

i.e.

$$
\left(A_{i} f\right)\left(j_{1}, \ldots, j_{i}, \ldots, j_{n}\right)=f\left(j_{1}, \ldots, j_{i}+1, \ldots, j_{n}\right)
$$

Then $A_{1}, A_{2}, \ldots, A_{n}$ are symmetric and pairwise commuting:

$$
\left\langle A_{i} f, g\right\rangle=\left\langle f, A_{i} g\right\rangle, \quad A_{i} A_{j}=A_{j} A_{i}, \quad f, g \in \mathscr{H}_{0} .
$$

Condition (ii) implies that

$$
a_{i} \cdot\|f\|^{2} \leq\left\langle A_{i} f, f\right\rangle \leq b_{i} \cdot\|f\|^{2}, \quad f \in \mathscr{K}_{0},
$$

where $\|\cdot\|$ is the norm derived from the inner product $\langle\cdot, \cdot\rangle$. These inequalities imply that the linear transformations $A_{1}, A_{2}, \ldots, A_{n}$ are bounded on $\mathscr{L}_{0}[12]$.

Let $\mathscr{H}$ be the completion of the inner-product space $\mathscr{H}_{0}$. Then $\mathscr{H}$ is a functional Hilbert space $[6,10]$ with reproducing kernel $K$ defined by

$$
K(j, k)=K_{j}(k)=c_{j+k}, \quad j, k \in N^{n},
$$

and the linear operators $B_{1}, B_{2}, \ldots, B_{n}$ defined by

$$
\left(B_{i} f\right)(j)=f\left(j+e_{i}\right), \quad f \in \mathscr{H}, j \in N^{n},
$$


are the unique continuous extensions of the linear transformations $A_{1}, A_{2}, \ldots, A_{n}$, respectively, to $\mathscr{H}$. The operators $B_{1}, B_{2}, \ldots, B_{n}$ are self-adjoint, pairwise commuting, and satisfy the inequalities

$$
a_{i} \cdot\|f\|^{2} \leq\left\langle B_{i} f, f\right\rangle \leq b_{i} \cdot\|f\|^{2}, \quad f \in \mathscr{H} .
$$

By the spectral theorem for finite pairwise-commuting family of self-adjoint operators $[8,12,14]$, there exists a resolution of the identity $E$ defined on the Borel subsets of $R^{n}$ such that

$$
\left\langle B_{i} f, g\right\rangle=\int_{[a, b]} x_{i} d\langle E(x) f, g\rangle, \quad f, g \in \mathscr{H},
$$

for $i=1,2, \ldots, n$. Define $\mu$ on the Borel subsets $S$ of $R^{n}$ by $\mu(S)=\left\langle E(S) K_{0}, K_{0}\right\rangle$. Then $\mu$ is a finite positive Borel measure on $R^{n}$, and

$$
\begin{aligned}
\int_{[a, b]} x^{j} d \mu(x) & =\int_{[a, b]} x_{1}^{j_{1}} x_{2}^{j_{2}} \cdots x_{n}^{j_{n}} d \mu\left(x_{1}, x_{2}, \ldots, x_{n}\right) \\
& =\left\langle B_{1}^{j_{1}} B_{2}^{j_{2}} \cdots B_{n}^{j_{n}} K_{0}, K_{0}\right\rangle \\
& =\left(B_{1}^{j_{1}} B_{2}^{j_{2}} \cdots B_{n}^{j_{n}} K_{0}\right)(0) \\
& =K_{0}\left(j_{1}, j_{2}, \ldots, j_{n}\right) \\
& =c\left(j_{1}, j_{2}, \ldots, j_{n}\right)=c_{j}, \quad j \in N^{n} .
\end{aligned}
$$

Hence (2) holds.

Now we shall prove that $\mu$ is supported on $K$. Since $E([a, b])$ is the identity operator on $\mathscr{H}, \mu\left(R^{n}\right)=\mu([a, b])$, and hence $\mu$ is supported on $[a, b]$. Let $B\left(w, r_{w}\right)$ be any one of the "holes" of $K$. For every polynomial with complex coefficients, say

$$
P(x)=\sum \varsigma_{j} x^{j}=\sum \varsigma_{\left(j_{1}, j_{2}, \ldots, j_{n}\right)} x_{1}^{j_{1}} x_{2}^{j_{2}} \cdots x_{n}^{j_{n}}
$$

we have that .

$$
\begin{aligned}
\int_{[a, b]} & P_{w}(x)|P(x)|^{2} d \mu(x) \\
& =\int_{[a, b]}\left(\sum_{i=1}^{n}\left(x_{i}-w_{i}\right)^{2}-r_{w}^{2}\right) \cdot\left(\sum \varsigma_{j} \bar{\zeta}_{k} x^{j+k}\right) d \mu(x) \\
& =\sum c_{j+k}^{w} \zeta_{j} \bar{\zeta}_{k} \geq 0
\end{aligned}
$$

by (iii). To prove that $\mu$ puts no mass on $B\left(w, r_{w}\right)$, let $0<\varepsilon<r_{w}$, and let $f$ be a continuous function on $R^{n}$ such that

$$
\begin{array}{ll}
0 \leq f(x) \leq 1 & \text { if } x \in R^{n} \\
f(x)=0 & \text { if } x \notin B\left(w, r_{w}\right), \\
f(x)=1 & \text { if } x \in B(w, \varepsilon) .
\end{array}
$$

By the Stone-Weierstrass theorem, there exists a sequence of polynomials $\left\{P_{m}\right\}_{m=1}^{\infty}$ which converges uniformly to $f$ on $[a, b]$, and thus

$$
\int_{[a, b]} P_{w}(x)|f(x)|^{2} d \mu(x)=\lim _{m \rightarrow \infty} \int_{[a, b]} P_{w}(x)\left|P_{m}(x)\right|^{2} d \mu(x) \geq 0 .
$$


Since $P_{w}(x)<0$ for every $x$ in $B\left(w, r_{w}\right)$,

$$
\begin{aligned}
\int_{[a, b]} P_{w}(x)|f(x)|^{2} d \mu(x)= & \int_{B\left(w, r_{w}\right)} P_{w}(x)|f(x)|^{2} d \mu(x) \\
& \leq \int_{B(w, \varepsilon)} P_{w}(x)|f(x)|^{2} d \mu(x) \\
& \leq\left(\varepsilon^{2}-r_{w}^{2}\right) \cdot \int_{B(w, \varepsilon)}|f(x)|^{2} d \mu(x) \\
& =\left(\varepsilon^{2}-r_{w}^{2}\right) \cdot \mu(B(w, \varepsilon)),
\end{aligned}
$$

and therefore $\left(\varepsilon^{2}-r_{w}^{2}\right) \cdot \mu(B(w, \varepsilon)) \geq 0$. Since $0<\varepsilon<r_{w}$, we have that $\mu(B(w, \varepsilon))=$ 0 , and since $\varepsilon$ was arbitrary, $\mu\left(B\left(w, r_{w}\right)\right)=0$.

Since the family $\left\{B\left(w, r_{w}\right): W \in W\right\}$ is at most countable, $\mu$ puts no mass on its union and is therefore supported on $K$. Q.E.D.

2. In the one dimensional case, in which $K$ is a compact subset of the real line $R$ and $\left\{c_{j}\right\}_{j=0}^{\infty}$ is a sequence of real numbers, a specific decomposition of $K$ is available. We have only to set $a=\min K, b=\max K$, and to let $\left\{\left(\alpha_{l}, \beta_{l}\right): l \in L\right\}$ be the set of connected components $\left(\alpha_{l}, \beta_{l}\right)$ of the open set $[a, b] \backslash K ;\left\{\left(\alpha_{l}, B_{l}\right): l \in L\right\}$ is either empty, finite, or countably infinite. For each connected component $\left(\alpha_{l}, \beta_{l}\right)$ of $[a, b] \backslash K$, there is a corresponding polynomial $P_{l}(x)=\left(x-\alpha_{l}\right)\left(x-\beta_{l}\right)$ which is negative if and only if $\alpha_{l}<x<\beta_{l}$. In the notation of $\S 1,\left(\alpha_{l}, \beta_{l}\right)=B\left(w, r_{w}\right)=$ $\left\{x \in R:|x-w|<r_{w}\right\}$, where $w=w_{l}=\left(\alpha_{l}+\beta_{l}\right) / 2$ and $r_{w}=\left(\alpha_{l}-\beta_{l}\right) / 2$, and $P_{l}(x)=P_{w}(x)=|x-w|^{2}-r_{w}^{2}$. Thus we have the following one-dimensional version of Theorem 1.

THEOREM 2. Suppose that $K$ is a compact subset of the real line and that $\left\{c_{j}\right\}_{j=0}^{\infty}$ is a sequence of real numbers. Let $a=\min k, b=\max K$, and let $\left\{\left(\alpha_{l}, \beta_{l}\right): l \in L\right\}$ be the set of connected components of $[a, b] \backslash K$. Then there exists a positive Borel measure $\mu$ supported on $K$ such that

$$
c_{j}=\int_{K} x^{j} d \mu(x), \quad j=1,2, \ldots,
$$

if and only if, for every sequence of complex numbers $\left\{\zeta_{j}\right\}_{j=0}^{\infty}$ with only finitely many nonzero terms,

(i) $\sum c_{j+k} \zeta_{j} \bar{\zeta}_{k} \geq 0$,

(ii) $a \cdot \sum c_{j+k} \zeta_{j} \bar{\zeta}_{k} \leq \sum c_{j+k+1} \varsigma_{j} \bar{\zeta}_{k} \leq b \cdot \sum c_{j+k} \varsigma_{j} \bar{\zeta}_{k}$,

(iii) for every $l \in L, \sum\left(c_{j+k+2}-\left(\alpha_{l}+\beta_{l}\right) c_{j+k+1}+\alpha_{l} \beta_{l} c_{j+k}\right) \geq 0$.

This theorem is a corollary of a theorem of Kreln and Nudel'man [11, Theorem 2.2, p. 293], but its proof does not depend upon any theorems on the form of polynomials which are nonnegative on $K$ (see [11, Lemma 2.1, p. 292]). In the multidimensional case, such theorems on the form of polynomials nonnegative on $K$ are not available (see [3]).

3. Our methods can also be applied to the trigonometric moment problem for a compact subset of the unit circle. Let $C$ be the complex plane; let $T$ be the unit circle in $C, T=\{z \in C:|z|=1\}$; and let $K$ be a compact subset of $T$. For all real numbers $\alpha$ and $\beta$ with $\alpha<\beta$, let

$$
\operatorname{arc}(\alpha, \beta)=\left\{e^{i t} \in T: \alpha<t<\beta\right\} .
$$


Letting $\theta$ be any real number such that $e^{i \theta} \in K$, we have that the connected components of $T \backslash K$ are a family of open $\operatorname{arcs}\left\{\operatorname{arc}\left(\alpha_{l}, \beta_{l}\right): l \in L\right\}$, where $\theta \leq \alpha_{l}<$ $\beta_{l} \leq \theta+2 \pi$ for every $l \in L$; this family of open arcs is either empty, finite, or countably infinite. For each connected component $\operatorname{arc}\left(\alpha_{l}, \beta_{l}\right)$ of $T \backslash K$, there is a corresponding trigonometric polynomial

$$
\begin{aligned}
T_{l}(t) & =4 \sin \left(\frac{t-\alpha_{l}}{2}\right) \sin \left(\frac{t-\beta_{l}}{2}\right) \\
& =2 \cos \left(\frac{\alpha_{l}-\beta_{l}}{2}\right)-e^{-i\left(\alpha_{l}+\beta_{l}\right) / 2} e^{i t}-e^{i\left(\alpha_{l}+\beta_{l}\right) / 2} e^{-i t}
\end{aligned}
$$

$T_{l}(t)$ is negative if and only if $e^{i t} \in \operatorname{arc}\left(\alpha_{l}, \beta_{l}\right)$.

THEOREM 3. Suppose that $K$ is a compact subset of the unit circle $T$ and that $\left\{c_{j}\right\}_{j=-\infty}^{j=+\infty}$ is a sequence of complex numbers. Let $\left\{\operatorname{arc}\left(\alpha_{l}, \beta_{l}\right): l \in L\right\}$ be the family of connected components of $T \backslash K$, where $\theta \leq \alpha_{l}<\beta_{l} \leq \theta+2 \pi$ for every $l \in L$, for some real number $\theta$. Then there exists a positive Borel measure $\mu$ supported on $K$ such that

$$
c_{j}=\int_{K} z^{j} d \mu(z)=\int_{K} e^{i j t} d \mu\left(e^{i t}\right), \quad j=0, \pm 1, \pm 2, \ldots
$$

if and only if, for every sequence of complex numbers $\left\{\zeta_{j}\right\}_{j=-\infty}^{j=+\infty}$ with only finitely many nonzero terms,

(i) $\sum c_{j-k} \zeta_{j} \bar{\zeta}_{k} \geq 0$, and

(ii) for every $l \in L$,

$$
\sum\left(2 \cos \left(\frac{\alpha_{l}-\beta_{l}}{2}\right) c_{j-k}-e^{-i\left(\alpha_{l}+\beta_{l}\right) / 2} c_{j-k+1}-e^{i\left(\alpha_{l}+\beta_{l}\right) / 2} c_{j-k-1}\right) \zeta_{j} \bar{\zeta}_{k} \geq 0
$$

The proof of this theorem is similar to that of Theorem 2, except that the spectral theorem for unitary operators is used instead of the spectral theorem for selfadjoint operators. Again, the idea of using the trigonometric polynomials $T_{l}$ to describe the "holes" of $K$ as derived from [11], where a stronger theorem is proved (Theorem 2.3 , p. 295), but the proof of Theorem 3 does not depend on any theorems on the form of trigonometric polynomials which are nonnegative on $K$ (see [11, Lemma 2.2, p. 294]).

\section{REFERENCES}

1. N. I. Akhiezer, The classical moment problem and some related questions in analysis, Oliver \& Boyd, Edinburgh and London, 1965.

2. C. Berg, The multidimensional moment problem and semigroups, Moments in Mathematics (H. J. Landau, ed.), Proc. Sympos. Appl. Math., vol. 37, Amer. Math. Soc., Providence, R.I., 1987.

3. C. Berg, J. P. R. Christensen and C. U. Jensen, A remark on the multidimensional moment problem. Math. Ann. 243 (1979), 163-169.

4. C. Berg and P. H. Maserick, Polynomially positive definite sequences, Math. Ann. 259 (1982), 487-495.

5. G. Cassier, Problème des moments sur un compact de $\mathbf{R}^{n}$ et décomposition de polynômes à plusiers variables, J. Funct. Anal. 58 (1984), 254-266.

6. W. F. Donoghue, Jr., Monotone matrix functions and analytic continuation, Springer-Verlag, New York, Heidelberg, Berlin, 1974. 
7. V. A. Fil'stinskǐ, The power moment problem on the entire axis with a given finite number of empty intervals in the spectrum, Zap. Meh.-Mat. Fak. Har'kov. Gos. Univ. i Har'kov. Mat. Ovšc (4) 30 (1964), 186-200. MR 35\#676.

8. P. R. Halmos, Introduction to Hilbert space and the theory of spectral multiplicity, 2nd ed., Chelsea, New York, 1957.

9. F. Hausdorff, 'Summationsmethoden und Momentfolgen. I, II, Math. Z. 9 (1921), 74-109, 280299.

10. E. Hille, Introduction to the general theory of reproducing kernels, Rocky Mountain J. Math. 2 (1972), 321-368.

11. M. G. Kreǐn and A. A. Nudel'man, The Markov moment problem and extremal problems, Trans. Math. Monographs, vol. 50, Amer. Math. Soc., Providence, R.I., 1977.

12. M. H. Stone, Linear transformations in Hilbert space and their applications to analysis, Amer. Math. Soc. Colloq. Publ, vol. 15, New York, 1932.

13. __ The generalized Weierstrass approximation theorem, Math. Mag. 21 (1948), 167-184, 327-354.

14. B. von Sz.-Nagy, Spektraldarstellung linearer Transformationen des Hilbertschen Raumes, Springer-Verlag, Berlin, Heidelberg, New York, 1967.

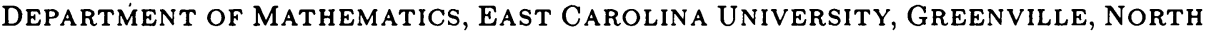
CAROLINA 27858-4353 